\title{
Estimating the effects of non-pharmaceutical interventions on COVID-19 in Europe
}

https://doi.org/10.1038/s41586-020-2405-7

Received: 30 March 2020

Accepted: 22 May 2020

Published online: 8 June 2020

Check for updates

\author{
Seth Flaxman ${ }^{1,7}$, Swapnil Mishra ${ }^{2,7}$, Axel Gandy ${ }^{1,7}$, H. Juliette T. Unwin ${ }^{2}$, Thomas A. Mellan ${ }^{2}$, \\ Helen Coupland ${ }^{2}$, Charles Whittaker ${ }^{2}$, Harrison Zhu', Tresnia Berah', Jeffrey W. Eaton', \\ Mélodie Monod', Imperial College COVID-19 Response Team*, Azra C. Ghani ${ }^{2}$, \\ Christl A. Donnelly,3, Steven Riley ${ }^{2}$, Michaela A. C. Vollmer ${ }^{2}$, Neil M. Ferguson ${ }^{2}$, Lucy C. Okell ${ }^{2}$ \\ \& Samir Bhatt ${ }^{2,7 凶}$
}

Following the detection of the new coronavirus ${ }^{1}$ severe acute respiratory syndrome coronavirus 2 (SARS-CoV-2) and its spread outside of China, Europe has experienced large epidemics of coronavirus disease 2019 (COVID-19). In response, many European countries have implemented non-pharmaceutical interventions, such as the closure of schools and national lockdowns. Here we study the effect of major interventions across 11 European countries for the period from the start of the COVID-19 epidemics in February 2020 until 4 May 2020, when lockdowns started to be lifted. Our model calculates backwards from observed deaths to estimate transmission that occurred several weeks previously, allowing for the time lag between infection and death. We use partial pooling of information between countries, with both individual and shared effects on the time-varying reproduction number $\left(R_{t}\right)$. Pooling allows for more information to be used, helps to overcome idiosyncrasies in the data and enables more-timely estimates. Our model relies on fixed estimates of some epidemiological parameters (such as the infection fatality rate), does not include importation or subnational variation and assumes that changes in $R_{t}$ are an immediate response to interventions rather than gradual changes in behaviour. Amidst the ongoing pandemic, we rely on death data that are incomplete, show systematic biases in reporting and are subject to future consolidation. We estimate that-for all of the countries we consider here-current interventions have been sufficient to drive $R_{t}$ below 1 (probability $R_{t}<1.0$ is greater than $99 \%$ ) and achieve control of the epidemic. We estimate that across all 11 countries combined, between 12 and 15 million individuals were infected with SARS-CoV-2 up to 4 May 2020, representing between $3.2 \%$ and $4.0 \%$ of the population. Our results show that major non-pharmaceutical interventions-and lockdowns in particular-have had a large effect on reducing transmission. Continued intervention should be considered to keep transmission of SARS-CoV-2 under control.
Following the identification of the new coronavirus SARS-CoV-2 in Wuhan (China) in December 2019 and its global spread, large epidemics of COVID-19 have ensued in Europe. In response to the rising numbers of cases and deaths and to preserve health systems, European countriesas with those in Asia-have implemented measures to control their epidemics. These large-scale non-pharmaceutical interventions vary between countries, but include social distancing (such as banning large gatherings), border closures, school closures, measures to isolate symptomatic individuals and their contacts, and large-scale lockdowns of populations with all but essential internal travel banned. Understanding whether these interventions have had the desired effect of controlling the epidemic, and which interventions are necessary to maintain control, is critical given their large economic and social costs. The key aim of these interventions is to reduce $R_{t}$, a fundamental epidemiological quantity that represents the average number of infections generated at time $t$ by each infected case over the course of their infection.

In China, strict movement restrictions and other measures (including case isolation and quarantine) began to be introduced from 23 January 2020, which achieved a downward trend in the number of confirmed new cases during February and resulted in zero new confirmed indigenous cases in Wuhan by 19 March 2020. Studies have estimated how the values of $R_{t}$ changed during this time in different

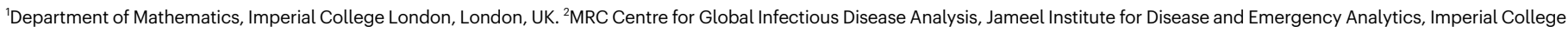

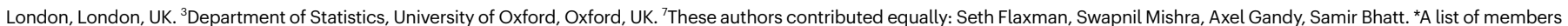
and their affiliations appears at the end of the paper. ${ }^{\circledR e}$-mail: s.bhatt@imperial.ac.uk 
areas of China, from around $2-4$ during the uncontrolled epidemic to below 1 (refs. ${ }^{1,2}$ ).

Estimating $R_{t}$ for SARS-CoV-2 presents challenges, owing to the high proportion of infections that are not detected by health systems $\mathrm{s}^{1,3,4}$ and to the regular changes in testing policies, which resulted in different proportions of infections being detected over time and between countries. Initially, most countries had the capacity to test only a small proportion of suspected cases and reserved tests for severely ill patients or for high-risk groups (for example, the contacts of positively tested individuals).

An alternative way to estimate the course of the epidemic is to calculate backwards from observed deaths to the number of infections. We introduce a Bayesian mechanistic model linking the infection cycle to observed deaths, inferring the total population infected (attack rates) as well as $R_{t}$. We assess whether there is evidence that interventions have so far been successful at reducing $R_{t}$ to values below 1 . We simulate a hypothetical counterfactual scenario in which $R_{t}$ remains at starting levels to estimate the deaths that would have occurred without interventions.

Reported deaths are likely to be far more reliable than case dataalthough reported death data still have limitations. First, early deaths attributable to COVID-19 may have been missed. Second, there is variation in the reporting of deaths by country and over time. Third, reporting delays are expected and can be both systematic and random in nature. We attempt to overcome these data limitations by using a consolidated data source, incorporating noise in our observational model, partially pooling of information between countries and performing a sensitivity analysis under scenarios of underreporting to test our conclusions (Supplementary Information).

Our model relies on fixed estimates of some epidemiological parameters, such as the onset-to-death distribution, the infection fatality rate and the generation distribution, that are based on previous work ${ }^{5,6}$; we perform a sensitivity analysis on these parameters. Our parametric form of $R_{t}$ assumes that changes in $R_{t}$ are an immediate response to interventions rather than gradual changes in behaviour, and it does not include importation or subnational variation. We assume that individual interventions have a similar effect in different countries, and that the efficacy of these interventions remains constant over time. Our framework infers $R_{t}$ from mortality data, while accounting for time lags since infections occurred. As a result, even with perfect data and partial pooling, we cannot perfectly predict the current value of $R_{t}$. However, the credible intervals on $R_{t}$ show the self-consistent behaviour that is a hallmark of a fully Bayesian analysis throughout the entire period we study, exhibiting appropriate shrinkage as more data become available (Supplementary Videos 1-3).

Italy was the first European country to begin major non-pharmaceutical interventions, and other countries followed soon afterwards (Extended Data Fig. 4). The onset of interventions ranged between 2 March and 29 March 2020 . We analysed data on mortality from COVID-19 in 11 European countries until 4 May 2020, at which point lockdowns were relaxed in Italy and Spain. For each country, we model the number of infections, the number of deaths and $R_{t}$ (Fig. 1 ). $R_{t}$ is modelled as a piecewise constant function that changes only when an intervention occurs. Each country has its own individual starting $R_{t}$ before interventions took place. For all countries, interventions are assumed to have the same relative effect on $R_{t}$ and are informed by mortality data across all countries. The only exception is that we use partial pooling to introduce country-specific effects of the effectiveness of the last intervention introduced in the study period in a country (which is usually lockdown).

\section{Estimated infections, $R_{t}$ and effect sizes}

In all countries, we estimate there are orders-of-magnitude fewer infections detected (Fig. 1, Extended Data Figs. 1,2) than true infections, most likely owing to mild and asymptomatic infections as well as limited testing capacities and changes in testing policy. In Italy, our results suggest that-cumulatively-2.8 (2.2-3.5) million people (all parenthetical ranges refer to $95 \%$ credible intervals) have been infected as of 4 May 2020, which gives an attack rate of $4.6 \%$ (3.6-5.8\%) of the population (Table 1). In Spain (which has also experienced a large number of deaths), we estimate that $5.5 \%$ of the population (2.6 (2.1-3.3) million people) have been infected to date. Germany, the most populous country in our study, is estimated to have one of the lowest attack rates at $0.85 \%$ with $710,000(550,000-930,000)$ people infected. Belgium has the highest estimated attack rates of $8 \%$, followed by Spain with $5.5 \%$. Although there have been few reliable national serological studies ${ }^{7}$, initial small-scale surveys in Austria ${ }^{8}$ and Denmark ${ }^{9}$ closely align with our estimates. A much larger study in Spain is very closely aligned with our estimates ${ }^{10}$. To some extent, these initial results validate our choice of infection fatality rate.

Averaged across all countries, we estimate the initial $R_{t}$ to be 3.8(2.45.6), consistent with previous analyses ${ }^{1,11}$. These estimates are informed by our choice of generation-interval distribution and the initial growth rate of observed deaths. A shorter assumed generation time results in lower starting $R_{t}$ (Supplementary Discussion 3). The initial values of $R_{t}$ are also uncertain, owing to (a) importation (rather than local transmission) being the dominant source of new infections in the early period of the epidemic and (b) possible under-ascertainment in deaths, particularly before testing became widespread. We perform sensitivity analyses around these parameters (Supplementary Discussions 10,11).

We estimate large reductions in $R_{t}$ in response to the combined non-pharmaceutical interventions. Our results-which are driven more by countries with advanced epidemics and larger numbers of deaths-suggest that these interventions have together had a substantial effect on transmission, as measured by changes in the estimated $R_{t}$. At the time of this study, we find current estimates of $R_{t}$ to range from a posterior mean of $0.44(0.26-0.61)$ for Norway to a posterior mean of $0.82(0.73-0.93)$ for Belgium, with an average of 0.66 across the 11 countries - an $82 \%$ reduction compared to the pre-intervention values. For all countries, we find that current interventions have been sufficient to drive $R_{t}$ below 1 (probability $R_{t}<1.0$ is greater than $99 \%$ across all countries we consider) and achieve control of the epidemic. These conclusions are corroborated by studies from individual countries-France ${ }^{12}$, Spain ${ }^{13}$, Germany ${ }^{14}$ and the UK $^{15}$ - over a similar period, which arrive at very similar estimates despite different methodologies and data. For example, a previous study ${ }^{12}$ estimates an $R_{t}$ of 0.67 for France using hospitalization records (we estimate 0.68 ); for Germany, the Robert Koch Institute reports $R_{t}$ of 0.76 using electronically notified cases ${ }^{14}$ (we estimate 0.71 ). The retrospective stability of our model (Supplementary Videos 1-3) is variable when the implementations of interventions are very dissimilar; an example of this is seen in Sweden, where interventions were dissimilar to other countries and led initially to large uncertainty. Our model uncertainty is also dependent on the magnitude of $R_{t}$; this occurs because infections are a nonlinear function of $R_{t}$ and are sensitive to small increases. Uncertainty shrinks greatly when $R_{t}$ is reduced. Examples of this effect are seen in all countries, but it is most pronounced in Belgium and France; these countries show large uncertainties in the number of infections in the early period of the epidemic. Our choice of parameterizing $R_{t}$ using piecewise constant functions means that we cannot capture the fine-scale variation that could be achieved by using additional covariates.

Lockdown has an identifiable large effect on transmission (81\% (75-87\%) reduction) (Fig. 2). The close spacing of interventions in time (Extended Data Fig. 4) means that the individual effects of the other interventions are not identifiable (Fig. 2). Our partial pooling model requires only one country to provide a signal for the effect of a given intervention, and this effect is then shared across all countries. Although this sharing can potentially lead to initial over- or under-estimation of the effect of an intervention, it also means that a 

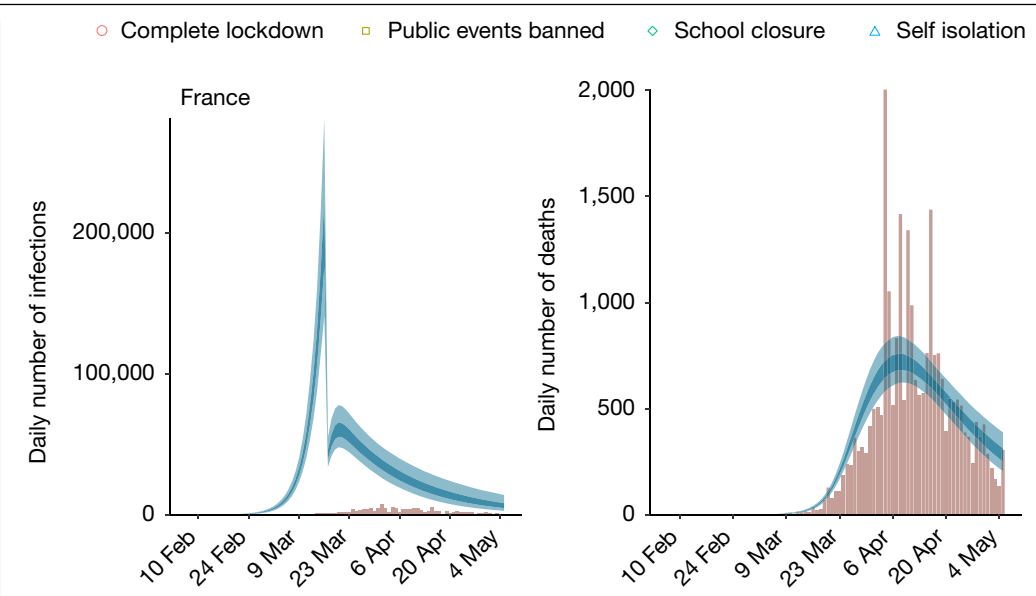

$\checkmark$ Social distancing

$50 \%$

$95 \%$
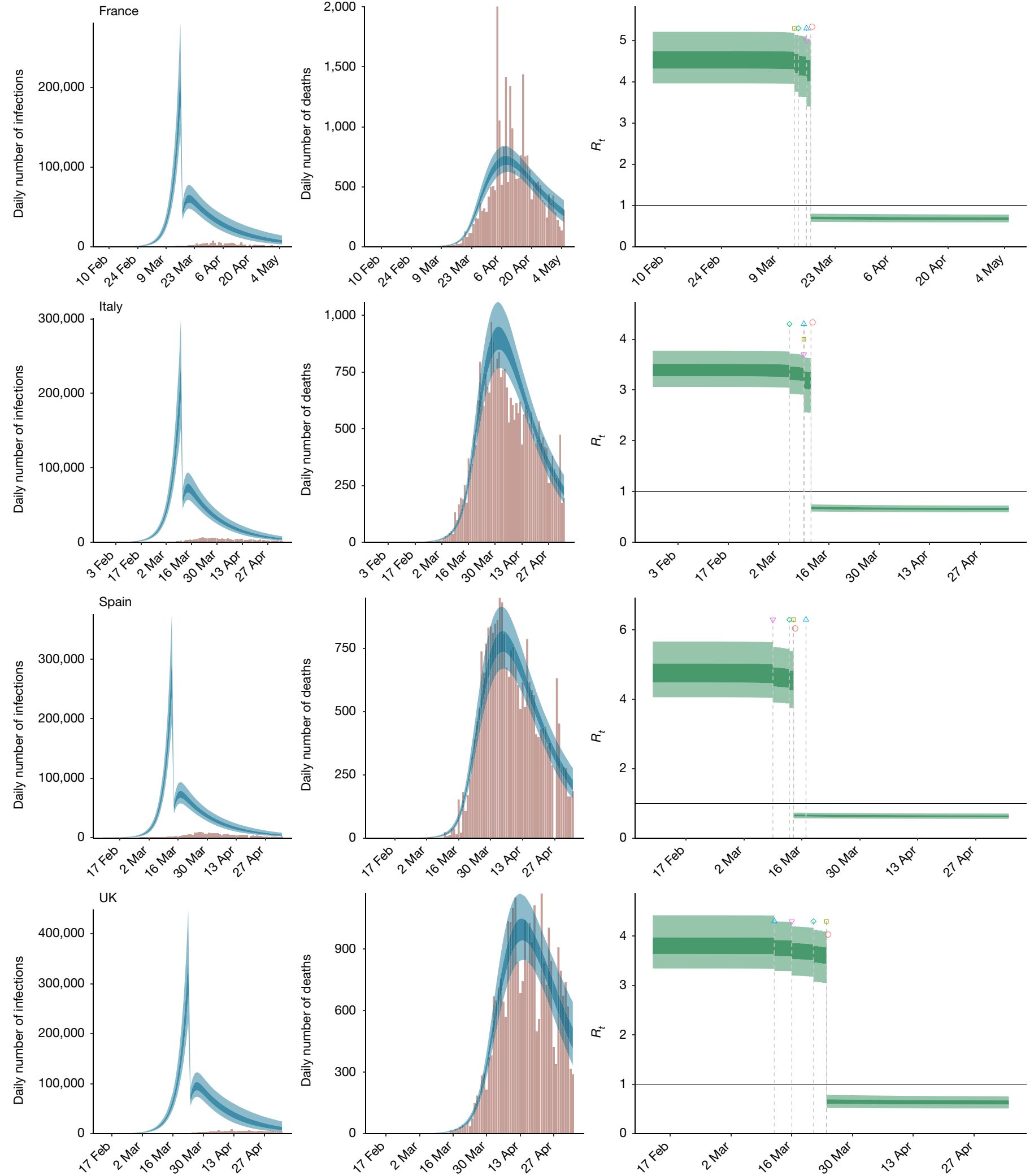

Fig. 1 Country-level estimates of infections, deaths and $\boldsymbol{R}_{\boldsymbol{t}}$ for France, Italy, Spain and the UK. Left, daily number of infections. Brown bars are reported infections; blue bands are predicted infections; dark blue, $50 \%$ credible interval; light blue $95 \%$ credible interval. The number of daily infections estimated by our model drops immediately after an intervention, as we assume that all infected people become immediately less infectious through the

intervention. Afterwards, if $R_{t}$ is above 1 , the number of infections will start growing again. Middle, daily number of deaths. Brown bars are reported deaths; blue bands are predicted deaths; credible intervals are as in the left plot. Right, $R_{t}$. Dark green, $50 \%$ credible interval; light green, $95 \%$ credible interval. Icons are interventions, shown at the time at which they occurred. 


\section{Table 1 | Total population infected by country}

\begin{tabular}{ll}
\hline Country & $\begin{array}{l}\text { Percentage of total population infected (mean (95\% credible } \\
\text { interval)) }\end{array}$ \\
\hline Austria & $0.76 \%(0.59-0.98 \%)$ \\
\hline Belgium & $8 \%(6.1-11 \%)$ \\
\hline Denmark & $1.0 \%(0.81-1.4 \%)$ \\
\hline France & $3.4 \%(2.7-4.3 \%)$ \\
\hline Germany & $0.85 \%(0.66-1.1 \%)$ \\
\hline Italy & $4.6 \%(3.6-5.8 \%)$ \\
\hline Norway & $0.46 \%(0.34-0.61 \%)$ \\
\hline Spain & $5.5 \%(4.4-7.0 \%)$ \\
\hline Sweden & $3.7 \%(2.8-5.1 \%)$ \\
\hline Switzerland & $1.9 \%(1.5-2.4 \%)$ \\
\hline UK & $5.1 \%(4.0-6.5 \%)$ \\
\hline
\end{tabular}

Posterior model estimates of the attack rate by country (percentage of total population infected) as of 4 May 2020. Results are derived from a model representing 11 countries with a total population of 375 million and 128,928 reported COVID-19-related deaths up to 4 May 2020.

consistent signal for all countries can be estimated before that signal is present in data from an individual country ${ }^{16}$. Therefore, this sharing is potentially useful for generating early warnings, by leveraging what happened in countries with earlier epidemics to inform countries with more-recent epidemics.

\section{Estimated effect of interventions on deaths}

Extended Data Table 1 shows total deaths forecast from the beginning of the epidemic up to and including 4 May 2020 under our fitted model and under the counterfactual model, which predicts what would have happened if no interventions were implemented (and $R_{t}=R_{0}$; that is, the initial $R_{t}$ estimated before interventions came into effect).

By comparing the deaths predicted under the model with no interventions to the deaths predicted in our intervention model, we calculated the total deaths averted in our study period. We find that across 11 countries $3.1(2.8-3.5)$ million deaths have been averted owing to interventions since the beginning of the epidemic; Extended Data Fig. 5 compares the actual total deaths to the counterfactual total deaths. The counterfactual model without interventions is illustrative only, and reflects the assumptions of our model. We do not account for changes in behaviour; in reality, even in the absence of government interventions we would expect $R_{t}$ to decrease and therefore would overestimate deaths in the no-intervention model. Conversely, we do not consider the effect on the infection fatality rate as a result of an overwhelmed health system in which patients may not be able to access critical care facilities, which would underestimate the number of counterfactual deaths. In the Supplementary Information, we show further counterfactual estimates under differing assumptions of the generation distribution and onset-to-death distribution and all scenarios broadly show the same trends. Given this agreement across differing scenarios, we believe our estimates for the counterfactual deaths averted to be plausible.

\section{Discussion}

During the ongoing transmission of SARS-CoV-2 in Europe, we analyse trends in the numbers of deaths to assess the extent to which transmission has been reduced. Representing the infection process associated with COVID-19 using a semi-mechanistic, joint Bayesian hierarchical model, we can reproduce trends observed in the data relating to deaths and produce empirically driven predictions that are valid over short time horizons.

We estimate that there have been many more infections than are currently reported. The high level of under-ascertainment of infections $\phi$ First intervention $\phi$ Later intervention

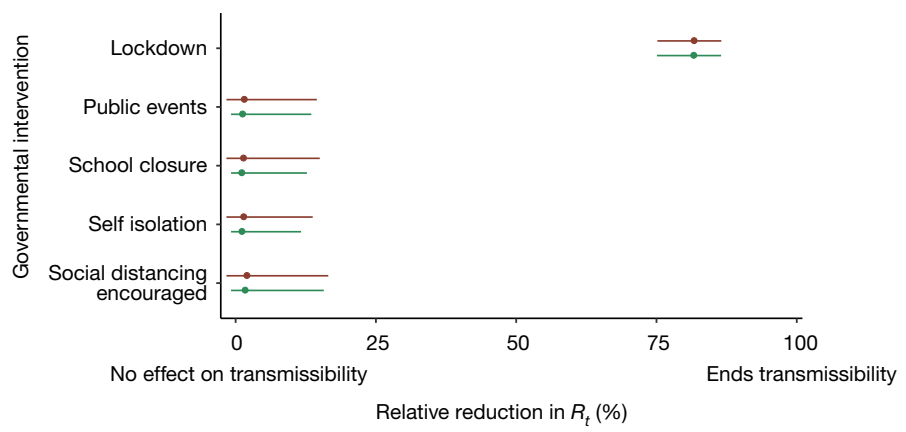

Fig. 2 | Effectiveness of interventions on $\boldsymbol{R}_{\boldsymbol{t}}$. Our model includes five covariates for governmental interventions, adjusting for whether the intervention was the first one undertaken by the government in response to COVID-19 (red) or was subsequent to other interventions (green). Mean relative percentage reduction in $R_{t}$ is shown with $95 \%$ posterior credible intervals. If $100 \%$ reduction is achieved, $R_{t}=0$ and there is no more transmission of COVID-19. Lockdown is significantly different from the other interventions; the other interventions are not significantly different from each other, probably owing to the fact that many interventions occurred on the same day or within days of each other (as shown in Extended Data Fig. 4). Results are derived from a model that represents 11 countries with a total population of 375 million and 128,928 reported COVID-19-related deaths up to 4 May 2020.

that we estimate here is probably due to the focus on testing in hospital settings, which misses milder or asymptomatic cases in the community. Despite this, we estimate that only a relatively small minority of individuals in each country have been infected (Table1). Our estimates imply that the populations in Europe are not close to herd immunity (about 70\% if $R_{0}$ is 3.8) $)^{17}$. Furthermore, with values of $R_{t}$ below 1 in all countries, the rate of acquisition of herd immunity will slow down rapidly. Our estimates for attack rates during our study period are consistent with those reported from national serological studies ${ }^{7}$. Similarly, comparable studies estimating $R_{t}$ all agree that the number as of 4 May 2020 is less than 1.

To our knowledge, our modelling approach is unique in pooling information from multiple countries at once. Using this approach means that we require a central consolidated data source (such as data from the European Centre of Disease Control (ECDC)), and also that trends in some countries will be affected by those countries with more data. We argue that this effect is beneficial, in that it helps to minimize idiosyncrasies in the data ${ }^{16}$, as well as to improve consistency of estimates over time. Although our qualitative conclusions surrounding the effect of interventions and the finding that $R_{t}$ is less than 1 are robust to our choice of whether to incorporate pooling or not, the ability to use a greater extent of available data and share information across countries in a statistically principled manner markedly improves the consistency of model predictions across the study period (Supplementary Videos 1-3).

Most interventions were implemented in rapid succession in many countries, and as such it is difficult to disentangle the individual effect sizes of each intervention. In our analysis, we find that only the effect of lockdown is identifiable, and that it has a substantial effect (81\% (75-87\%) reduction in $R_{t}$ ). Taking into account country-specific effects, the effect size of lockdown remains large across all countries (Supplementary Fig. 29).

We acknowledge the limitations of existing mortality data relating to COVID-19-in particular, deaths outside hospitals may be underreported. However, by using the ECDC data, we rely on a comprehensive data source that is refined and updated each day in a systematic process. Our sensitivity analysis of underreporting and statistical-measurement noise suggests that we may slightly 
underestimate the attack rates in some countries, but this does not change our overall conclusions pertaining to $R_{t}$. However, even if the data were complete, our method cannot surmount the time lag between infections and deaths and can only fully identify trends in infections 2-3 weeks earlier. Extensions of our model could use case, hospitalization or intensive care data, but reconciling the different biases inherent in these sources while ensuring parsimony is challenging and would require additional assumptions.

The modern understanding of infectious disease, combined with a global publicized response, has meant that nationwide interventions could be implemented with widespread adherence and support. Given the observed infection fatality ratios and the epidemiology of COVID-19, major non-pharmaceutical interventions have had an effect in reducing transmission in all of the countries we have considered. In all countries in this study, we find that these interventions have reduced $R_{t}$ below 1 , and have contained their epidemics at the current time. When looking at simplistic counterfactual models over the whole epidemic, the number of potential deaths averted is substantial. We cannot say for certain that the current measures will continue to control the epidemic in Europe; however, if current trends continue there is reason for optimism.

\section{Online content}

Any methods, additional references, Nature Research reporting summaries, source data, extended data, supplementary information, acknowledgements, peer review information; details of author contributions and competing interests; and statements of data and code availability are available at https://doi.org/10.1038/s41586-020-2405-7.

1. Li, R. et al. Substantial undocumented infection facilitates the rapid dissemination of novel coronavirus (SARS-CoV-2). Science 368, 489-493 (2020).

2. Zhang, J. et al. Patterns of human social contact and contact with animals in Shanghai, China. Sci. Rep. 9, 15141 (2019).

3. Zhao, A. J. et al. Antibody responses to SARS-CoV-2 in patients of novel coronavirus disease 2019. Clin. Infect. Dis. ciaa344 https://doi.org/10.1093/cid/ciaa344 (2020).

4. Jombart, T. et al. Inferring the number of COVID-19 cases from recently reported deaths. Wellcome Open Research 5, 78 (2020).

5. Verity, R. et al. Estimates of the severity of coronavirus disease 2019: a model-based analysis. Lancet 20, 669-677 (2020).

6. $\mathrm{Bi}$, Q. et al. Epidemiology and transmission of COVID-19 in 391 cases and 1286 of their close contacts in Shenzhen, China: a retrospective cohort study. Lancet Infect. Dis. https://doi.org/10.1016/S1473-3099(20)30287-5 (2020).
7. Bobrovitz, N. et al. Lessons from a rapid systematic review of early SARS-CoV-2 serosurveys. Preprint at medRxiv https://doi.org/10.1101/2020.05.10.20097451 (2020)

8. Statistics Austria. COVID-19 Prevalence Study: Maximum 0.15\% of Austrian Population Infected with SARS-CoV-2 (Statistics Austria, 2020).

9. Erikstrup, C. et al. Estimation of SARS-CoV-2 infection fatality rate by real-time antibody screening of blood donors. Clin. Infect. Dis. ciaa849 https://doi.org/10.1093/cid/ciaa849 (2020).

10. Pollán, M. et al. Prevalence of SARS-CoV-2 in Spain (ENE-COVID): a nationwide, population-based seroepidemiological study. Lancet https://doi.org/10.1016/S01406736(20)31483-5 (2020).

11. Zhang, J. et al. Age profile of susceptibility, mixing, and social distancing shape the dynamics of the novel coronavirus disease 2019 outbreak in China. Preprint at medRxiv https://doi.org/10.1101/2020.03.19.20039107 (2020).

12. Salje, H. et al. Estimating the burden of SARS-CoV-2 in France. Science 369, 208-211 (2020).

13. Hyafil, A. \& Morina, D. Analysis of the impact of lockdown on the reproduction number of the SARS-Cov-2 in Spain. Gac. Sanit. https://doi.org/10.1016/j.gaceta.2020.05.003 (2020).

14. Robert Koch Institute. Coronavirus Disease 2019 (COVID-19) Daily Situation Report of the Robert Koch Institute (Robert Koch Institute, 2020).

15. Davies, N. G., Kucharski, A. J., Eggo, R. M., Gimma, A. \& Edmunds, W. J. The effect of non-pharmaceutical interventions on COVID-19 cases, deaths and demand for hospital services in the UK: a modelling study. Lancet 5, E375-E385 (2020).

16. Gelman, A. \& Hill, J. Data Analysis using Regression and Multilevel/Hierarchical Models (Cambridge Univ. Press, 2006).

17. Miller, J. C. A note on the derivation of epidemic final sizes. Bull. Math. Biol. 74, 2125-2141 (2012).

Publisher's note Springer Nature remains neutral with regard to jurisdictional claims in published maps and institutional affiliations.

(c) The Author(s), under exclusive licence to Springer Nature Limited 2020

Imperial College COVID-19 Response Team

Pablo N. Perez-Guzman ${ }^{2}$, Nora Schmit ${ }^{2}$, Lucia Cilloni' ${ }^{2}$, Kylie E. C. Ainslie ${ }^{2}$, Marc Baguelin ${ }^{2}$ Adhiratha Boonyasiri ${ }^{4}$, Olivia Boyd ${ }^{2}$, Lorenzo Cattarino ${ }^{2}$, Laura V. Cooper ${ }^{2}$, Zulma Cucunubá2 ${ }^{2}$, Gina Cuomo-Dannenburg ${ }^{2}$, Amy Dighe² ${ }^{2}$, Bimandra Djaafara² Ilaria Dorigatti ${ }^{2}$, Sabine L. van Elsland ${ }^{2}$, Richard G. FitzJohn ${ }^{2}$, Katy A. M. Gaythorpe ${ }^{2}$, Lily Geidelberg ${ }^{2}$, Nicholas C. Grassly ${ }^{2}$, William D. Green ${ }^{2}$, Timothy Hallett ${ }^{2}$, Arran Hamlet ${ }^{2}$, Wes Hinsley' ${ }^{2}$, Ben Jeffrey², Edward Knock² ${ }^{2}$, Daniel J. Laydon ${ }^{2}$, Gemma Nedjati-Gilani ${ }^{2}$, Pierre Nouvellet ${ }^{2,5}$, Kris V. Parag ${ }^{2}$, Igor Siveroni ${ }^{2}$, Hayley A. Thompson ${ }^{2}$, Robert Verity ${ }^{2}$, Erik Volz ${ }^{2}$, Caroline E. Walters ${ }^{2}$, Haowei Wang ${ }^{2}$, Yuanrong Wang ${ }^{2}$, Oliver J. Watson ${ }^{2,6}$, Peter Winskill ${ }^{2}$, Xiaoyue Xi' \& Patrick G. T. Walker ${ }^{2}$

${ }^{4} \mathrm{NIHR}$ Health Protection Research Unit in Healthcare Associated Infections and Antimicrobial Resistance, Imperial College London, London, UK. ${ }^{5}$ School of Life Sciences, University of Sussex, Brighton, UK. ${ }^{6}$ Department of Laboratory Medicine and Pathology, Brown University, Providence, RI, USA. 


\section{Methods}

\section{Data}

Our model uses daily consolidated death data from the ECDC for 11 European countries currently experiencing the COVID-19 epidemic: Austria, Belgium, Denmark, France, Germany, Italy, Norway, Spain, Sweden, Switzerland and the UK. The ECDC provides information on confirmed cases and deaths attributable to COVID-19. For population counts, we use the United Nations Population Division age-stratified counts $^{18}$.

We also catalogue data on the nature and type of major non-pharmaceutical interventions. We looked at the government webpages from each country as well as their official public health webpages to identify the latest advice or laws being issued by the government and public health authorities. We collected the following: school closure ordered; case-based measures; public events banned; social distancing encouraged; lockdown decreed; and the time of the first and last intervention. A full list of the timing of these interventions and the sources we have used is provided in the Supplementary Notes, Supplementary Table 2.

By using the ECDC data, we rely on a consolidated data source compiled by the ECDC, who include many sources of data each day, constantly refining and updating data using a comprehensive and systematic process. However, despite the rigorous protocols, countries may vary in the specifics of the data that they report to the ECDC. For example, there is variation in reporting (that is, community versus hospital) and time lags. Despite these issues, we use ECDC data to ensure as much consistency as possible across all countries.

\section{Model}

A visual summary of our model is presented in Extended Data Fig. 3; details are provided in the Supplementary Methods.

We fit our model to observed deaths according to ECDC data from 11 European countries. The modelled deaths are informed by an infection-to-death distribution (Supplementary Fig. 1; derived from assumptions about the time from infection to the onset of symptoms and about the time from the onset of symptoms to death), and the population-averaged infection fatality ratio (adjusted for the age structure and contact patterns of each country, as discussed in the Supplementary Methods, Supplementary Table 3).

Given these distributions and ratios, modelled deaths are a function of the number of infections. The number of infections is modelled as the product of $R_{t}$ with a discrete convolution of the previous infections. Individual components of this convolution sum are weighted by the generation time distribution (the average time from the infection of one person to the time at which they infect another; Supplementary Fig. 2). In our work, we approximate the generation time distribution using the serial interval distribution. $R_{t}$ is a function of the initial $R_{t}$ before interventions and the effect sizes from interventions, in which interventions are modelled as piecewise constant functions.

Following the Bayesian hierarchy from bottom to top gives us a full framework to see how interventions affect infections, which can result in deaths. A schematic of our model is shown in Extended Data Fig. 3. To maximize the ability to observe the effect of interventions on deaths, we fit our model jointly for all 11 European countries, and use partial pooling of information between countries with both individual and shared effects on $R_{t}$. Partial pooling operates on the last intervention, which is-in most cases-lockdown. The effect of partial pooling can be seen in Supplementary Discussion 12, Supplementary Fig. 29. We chose a balanced prior that encodes the prior belief that interventions have an equal chance of having an effect or not, and ensure a uniform prior on the joint effect of all interventions (Supplementary Fig. 3). We evaluate the effect of our Bayesian prior distribution choices and evaluate our Bayesian posterior calibration to ensure our results are statistically robust.
We perform extensive model validation and sensitivity analyses. We validate our model by cross-validation over a 14-day period (Supplementary Discussion 1, Supplementary Table 1) and we show the fits for holdout samples in Supplementary Figs. 5-15. We check the convergence of the Markov chain Monte Carlo sampler (Supplementary Fig. 4). We consider the sensitivity of our estimates of $R_{t}$ to the mean of the generation distribution (Supplementary Discussion3, Supplementary Figs.16, 17). We further show that the choice of generation distribution does not change our counterfactual conclusions (Supplementary Fig. 18).Using univariate analyses and uninformative priors, we find (Supplementary Fig. 19) that all effects on their own serve to decrease $R_{t}$ (Supplementary Discussion 4). We compare our model to a non-parametric Gaussian Process model (Supplementary Discussion 5). To assess the effect of individual countries on the results, we perform a leave one country out' sensitivity analysis (Supplementary Discussion 6, Supplementary Figs. 20,21). To validate our starting values of $R_{t}$, we compare our model against an exponential-growth linear model (Supplementary Discussion 7, Supplementary Fig. 22). Instead of a joint analysis, we consider fits of our model to individual countries (Supplementary Discussion 8 , Supplementary Figs. 23-26). We perform a sensitivity analysis with respect to the onset-to-death distribution (Supplementary Discussion 9, Supplementary Fig. 27). We validate our probabilistic seeding scheme through an importance-sampling leave-one-out cross-validation (Supplementary Discussion 10). We consider a model extension with a constant, probabilistic under-reporting (Supplementary Discussion 11), finding that $R_{t}$ does not change substantially (Supplementary Fig. 28).

Our model is different to other approaches (such as EpiEstim ${ }^{19}$ ) that use the discrete renewal equation. We use the renewal equation as a latent process to model infections and propose a generative mechanism to connect these infections to death data. Simply applying the renewal equation directly to death data requires positing a mechanism in which deaths in the past can cause future deaths (see, for example, ref. ${ }^{20}$ ). In addition, for $R_{t}$, we are able to use a functional relationship in which non-pharmaceutical interventions can have a direct effect on $R_{t}$.

\section{Reporting summary}

Further information on research design is available in the Nature Research Reporting Summary linked to this paper.

\section{Data availability}

Death counts for the 11 European countries for the time period in our study and the full set of posterior draws from our model are available at https://reshare.ukdataservice.ac.uk/854380/.

\section{Code availability}

All source code and data necessary for the replication of our results and figures are available at https://github.com/ImperialCollegeLondon/covid19model. An R package based on our method is available at https://imperialcollegelondon.github.io/epidemia/.

18. United Nations, Department of Economic and Social Affairs, Population Division. World Population Prospects 2019: Data Booklet, ST/ESA/SER.A/424. (United Nations, 2019).

19. Cori, A., Ferguson, N. M., Fraser, C. \& Cauchemez, S. A new framework and software to estimate time-varying reproduction numbers during epidemics. Am. J. Epidemiol. 178, 1505-1512 (2013).

20. Goldstein, E. et al. Reconstructing influenza incidence by deconvolution of daily mortality time series. Proc. Natl Acad. Sci. USA 106, 21825-21829 (2009).

Acknowledgements S.B. acknowledges the NIHR BRC Imperial College NHS Trust Infection and COVID themes, the Academy of Medical Sciences Springboard award and the Bill and Melinda Gates Foundation. L.C.O. acknowledges funding from a UK Royal Society fellowship. Initial research on covariates in Supplementary Table 2 was crowdsourced; we thank a number of people across the world for help with this. This work was supported by Centre funding from the UK Medical Research Council under a concordat with the UK Department for International Development, the NIHR Health Protection Research Unit in Modelling Methodology and Community Jameel. We thank F. Valka for creating our website, and A. Gelman and the Stan 
team for helpful discussions. We acknowledge the resources provided by Cirrus UK National Tier-2 HPC Service at EPCC (http://www.cirrus.ac.uk) funded by the University of Edinburgh and EPSRC (EP/PO20267/1), and cloud compute time donated by Microsoft and Amazon.

Author contributions S.B., S.F., S.M. and A.G. conceived and designed the study. S.B., S.F., S.M., A.G., H.C., H.J.T.U., T.A.M., M.A.C.V., J.W.E. and N.M.F. performed analysis. L.C.O., S.B., S.F., A.G., A.C.G., C.A.D., S.R. and N.M.F. wrote the first draft of the paper. S.B., S.F., H.C., C.W., P.W., T.B., P.N.P.G., N.S., L. Cilloni, M.A.C.V. and H.C. collected data. All authors discussed the results and contributed to the revision of the final manuscript.
Competing interests The authors declare no competing interests.

Additional information

Supplementary information is available for this paper at https://doi.org/10.1038/s41586-0202405-7.

Correspondence and requests for materials should be addressed to S.B.

Peer review information Nature thanks David Earn and the other, anonymous, reviewer(s) for their contribution to the peer review of this work. Peer reviewer reports are available. Reprints and permissions information is available at http://www.nature.com/reprints. 

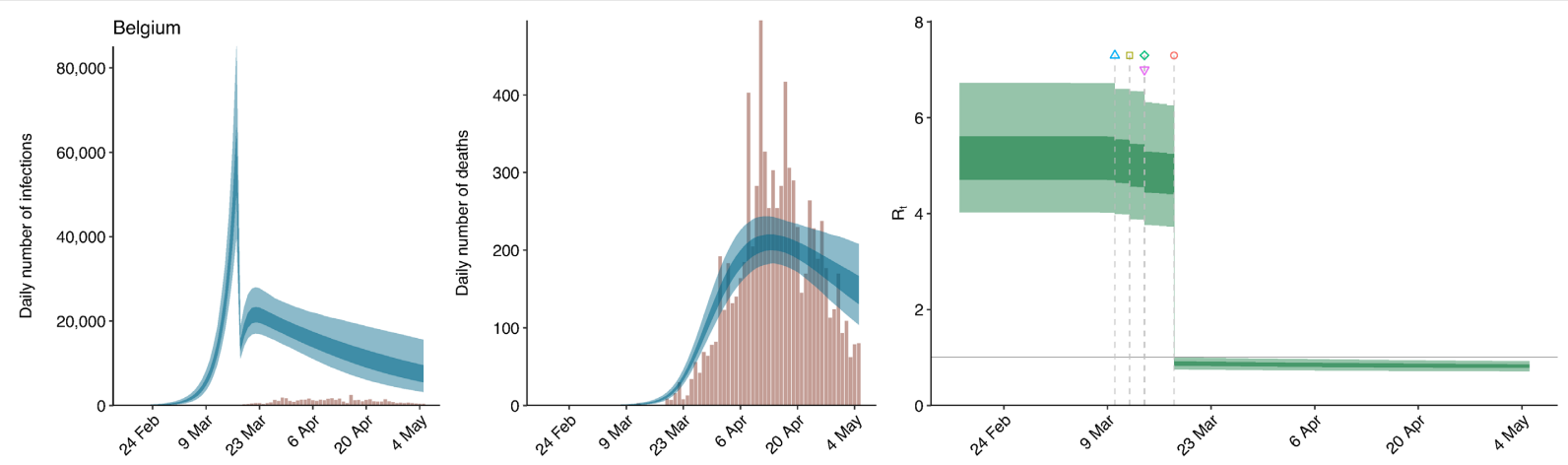

Interventions

Complete lockdown

- Public events banne

School closure

Self isolation

Social distancing

$50 \%$

$95 \%$
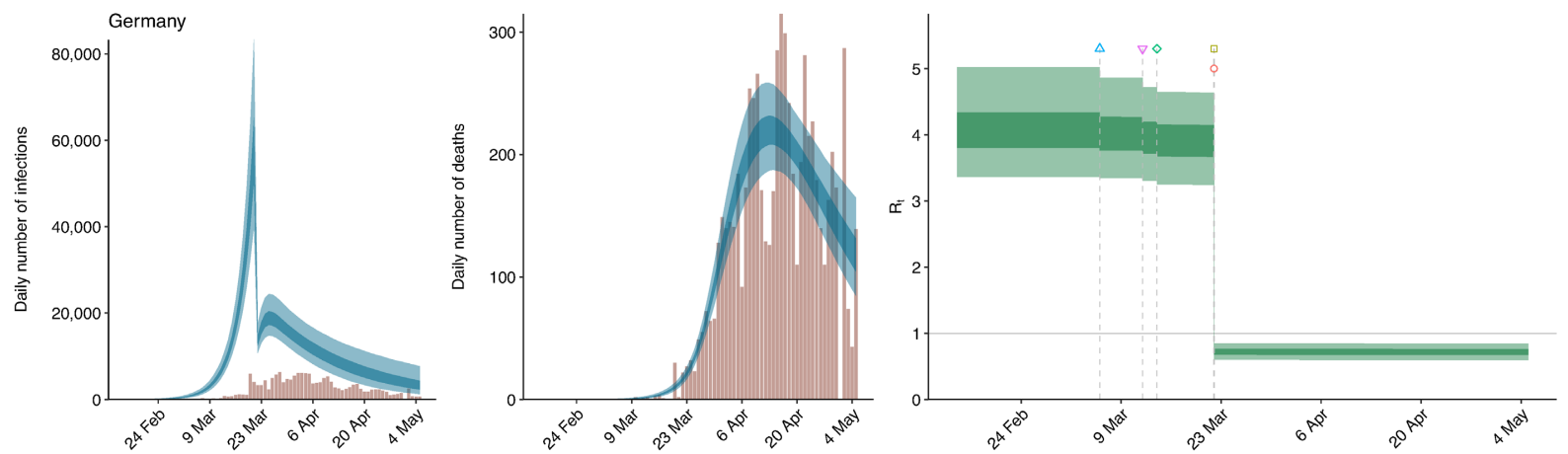

Interventions

Complete lockdown

Public events banned

- School closure

$\Delta$ Self isolation

Social distancing

$50 \%$

$95 \%$
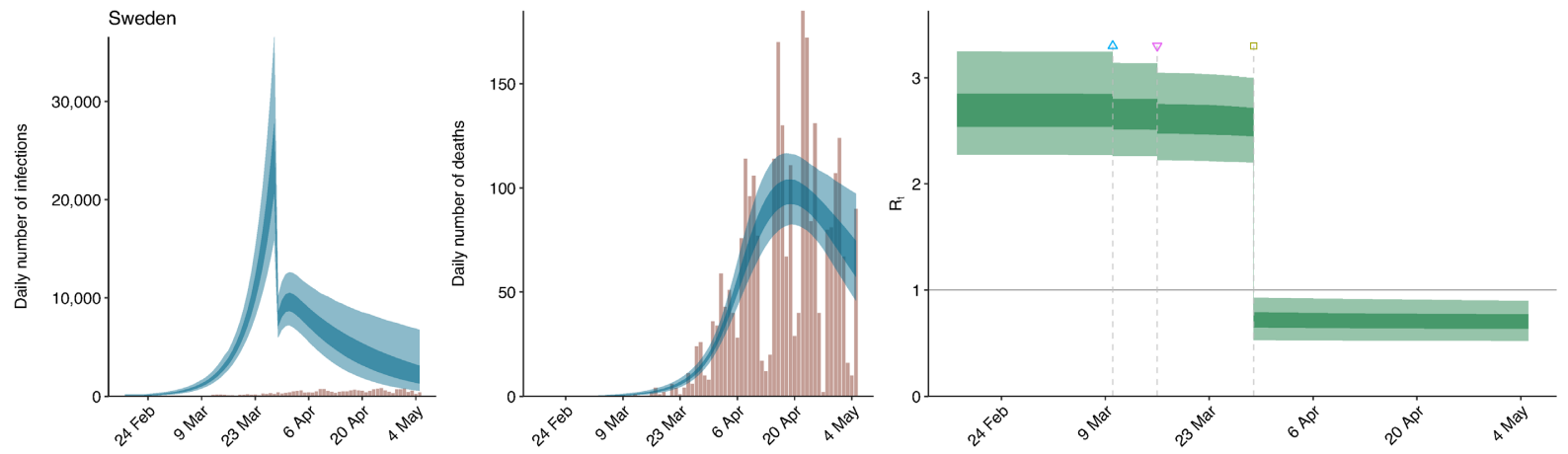

Interventions

Complete lockdown

Public events banne

School closure

Self isolation

Social distancing

$50 \%$
$95 \%$

$95 \%$
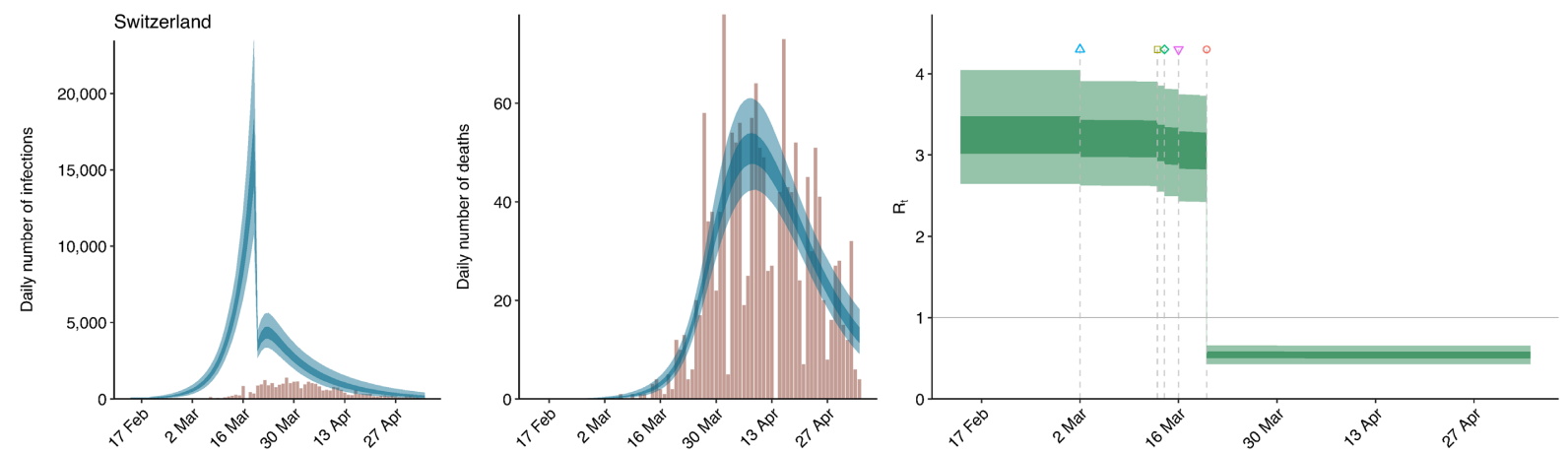

Interventions

Complete lockdown

Public events banne

Public events ba

School closu
Self isolation

Self isolation

$50 \%$

$95 \%$

Extended Data Fig. 1 | Country-level estimates of infections, deaths and $\boldsymbol{R}_{\boldsymbol{t}}$ for Belgium, Germany, Sweden and Switzerland. Left, daily number of infections. Brown bars are reported infections; blue bands are predicted infections; dark blue, 50\% credible interval; light blue, $95 \%$ credible interval. The number of daily infections estimated by our model drops immediately after an intervention, as we assume that all infected people become immediately less infectious through the intervention. Afterwards, if $R_{t}$ is above 1 , the number of infections will starts growing again. Middle, daily number of deaths. Brown bars are reported deaths; blue bands are predicted deaths; credible intervals are as in the left plot. Right, $R_{t}$. Dark green, $50 \%$ credible interval; light green, $95 \%$ credible interval. Icons are interventions, shown at the time at which they occurred. 

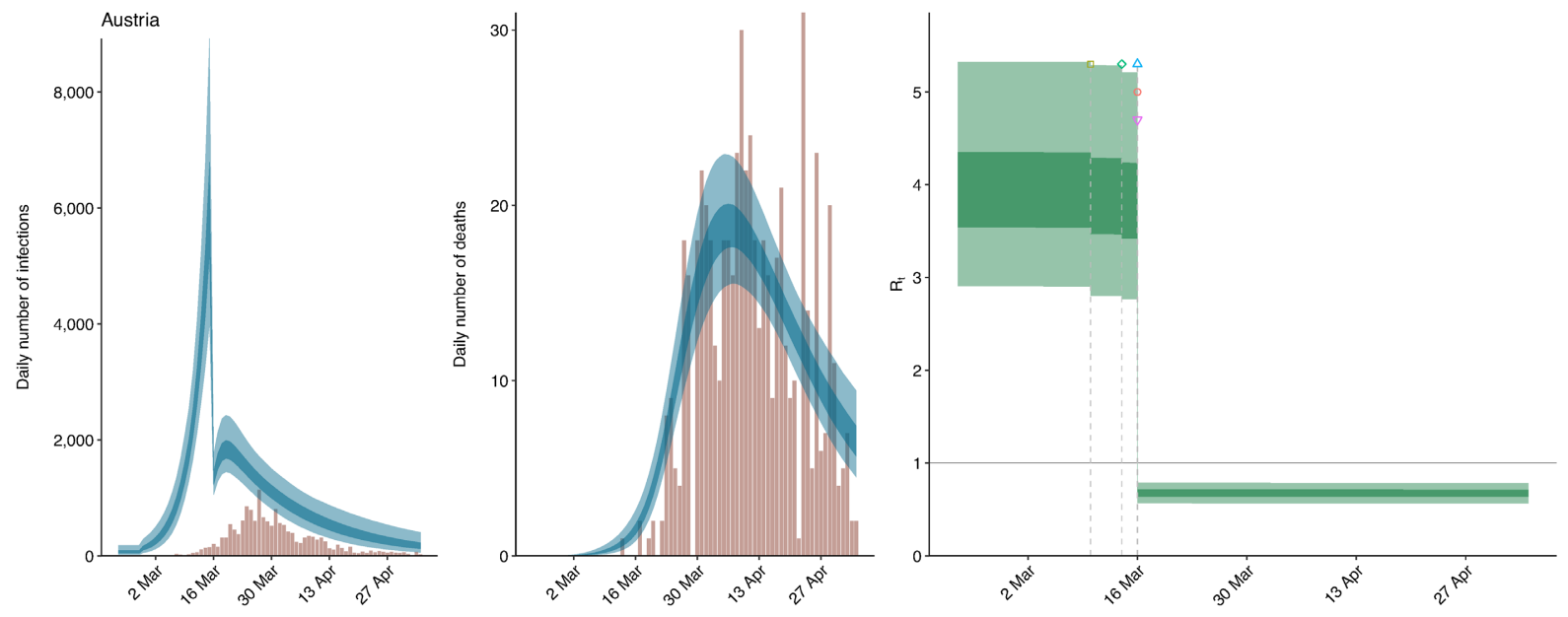

Interventions

Complete lockdown

Public events banne

- School closure

Self isolation

Social distancing
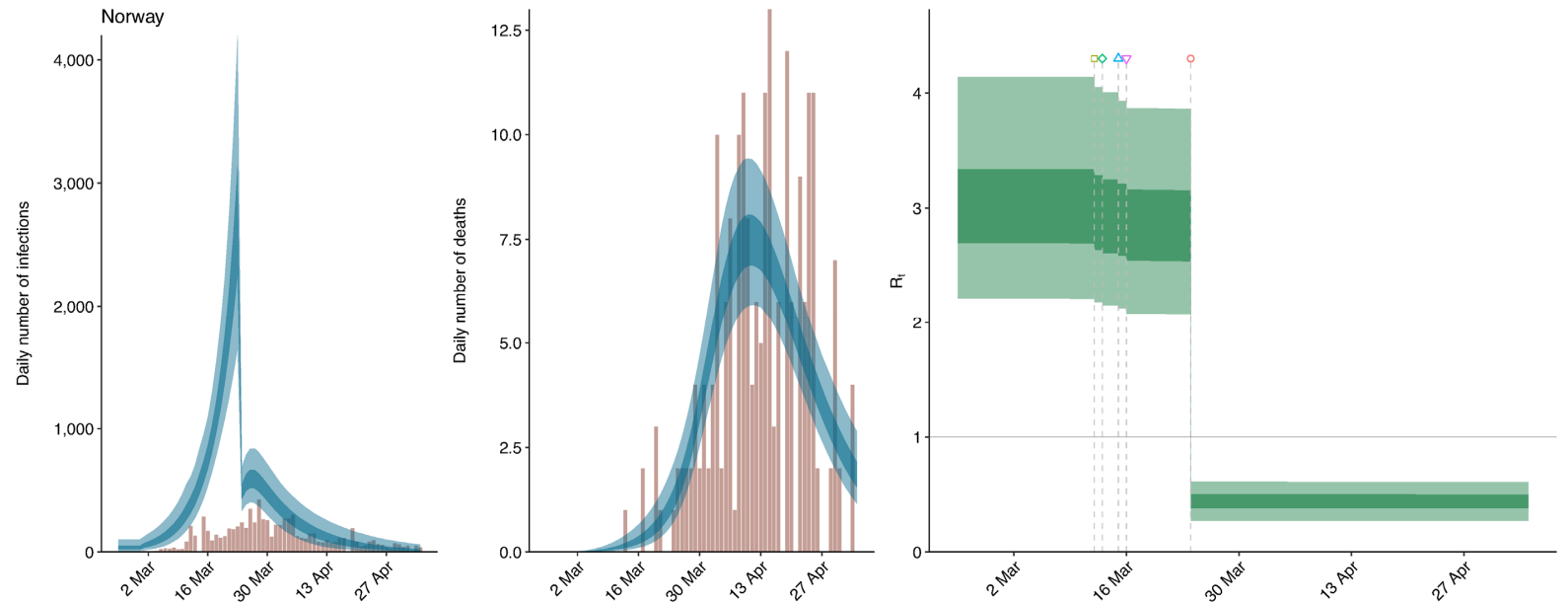

$50 \%$

$95 \%$

Interventions

Complete lockdown

Public events banne

School closure

Selfi isolation

$50 \%$
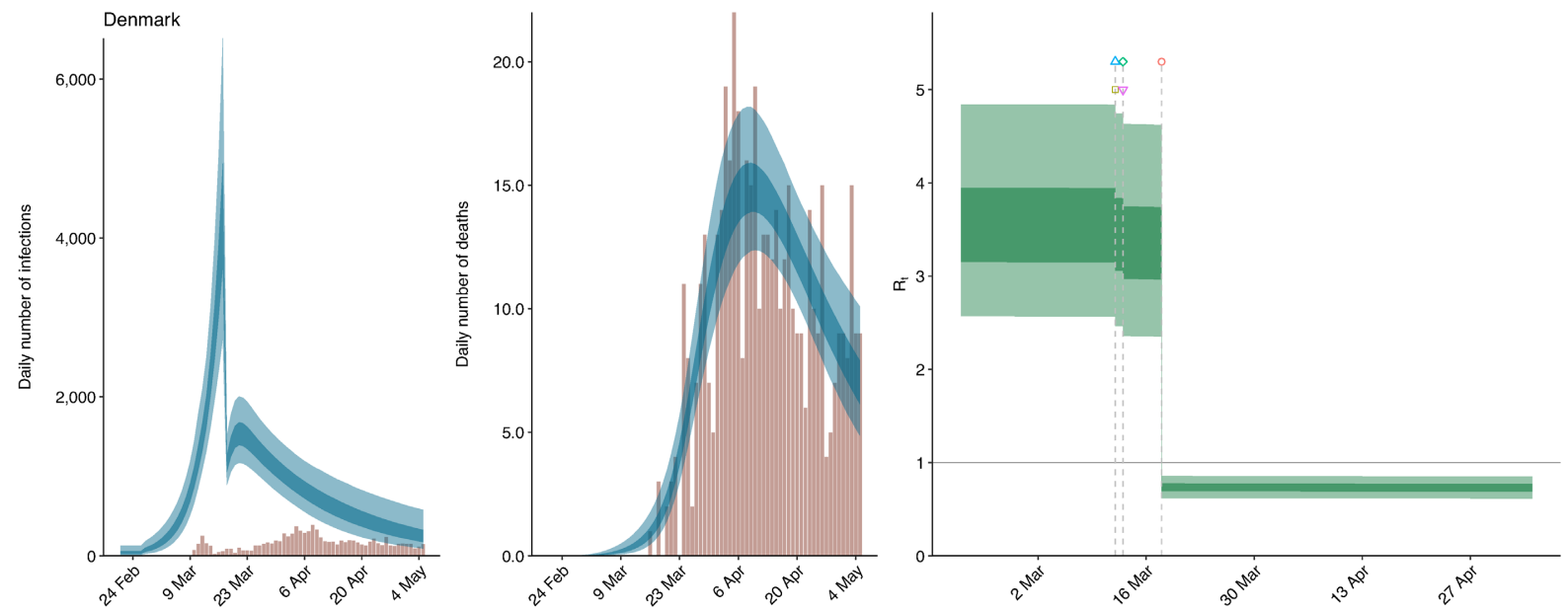

terventions

Complete lockdown Public events banned

School closure

Self isolation

Social distancing
Extended Data Fig. 2 | Country-level estimates of infections, deaths and $\boldsymbol{R}_{\boldsymbol{t}}$ for Austria, Norway and Denmark. Left, daily number of infections. Brown bars are reported infections; blue bands are predicted infections; dark blue, $50 \%$ credible interval; light blue, $95 \%$ credible interval. The number of daily infections estimated by our model drops immediately after an intervention, as we assume that all infected people become immediately less infectious through the intervention. Afterwards, if $R_{t}$ is above 1 , the number of infections will starts growing again. Middle, daily number of deaths. Brown bars are reported deaths; blue bands are predicted deaths; credible intervals are as in the left plot. Right, $R_{t}$. Dark green, $50 \% \mathrm{CI}$; light green, $95 \% \mathrm{CI}$. Icons are interventions, shown at the time at which they occurred. 


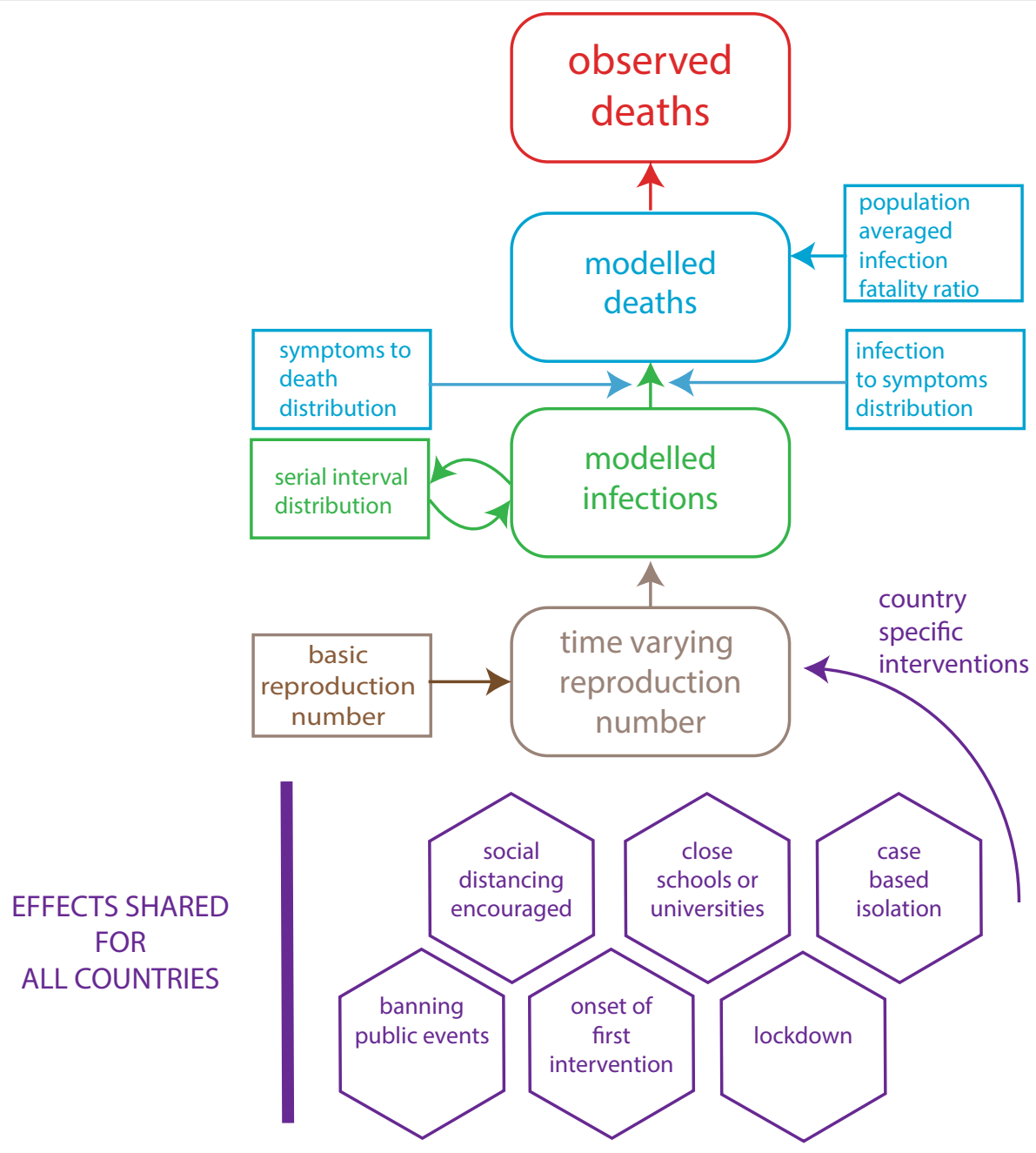

Extended Data Fig. 3 | Model summary. This figure shows a summary of the components of our model. 


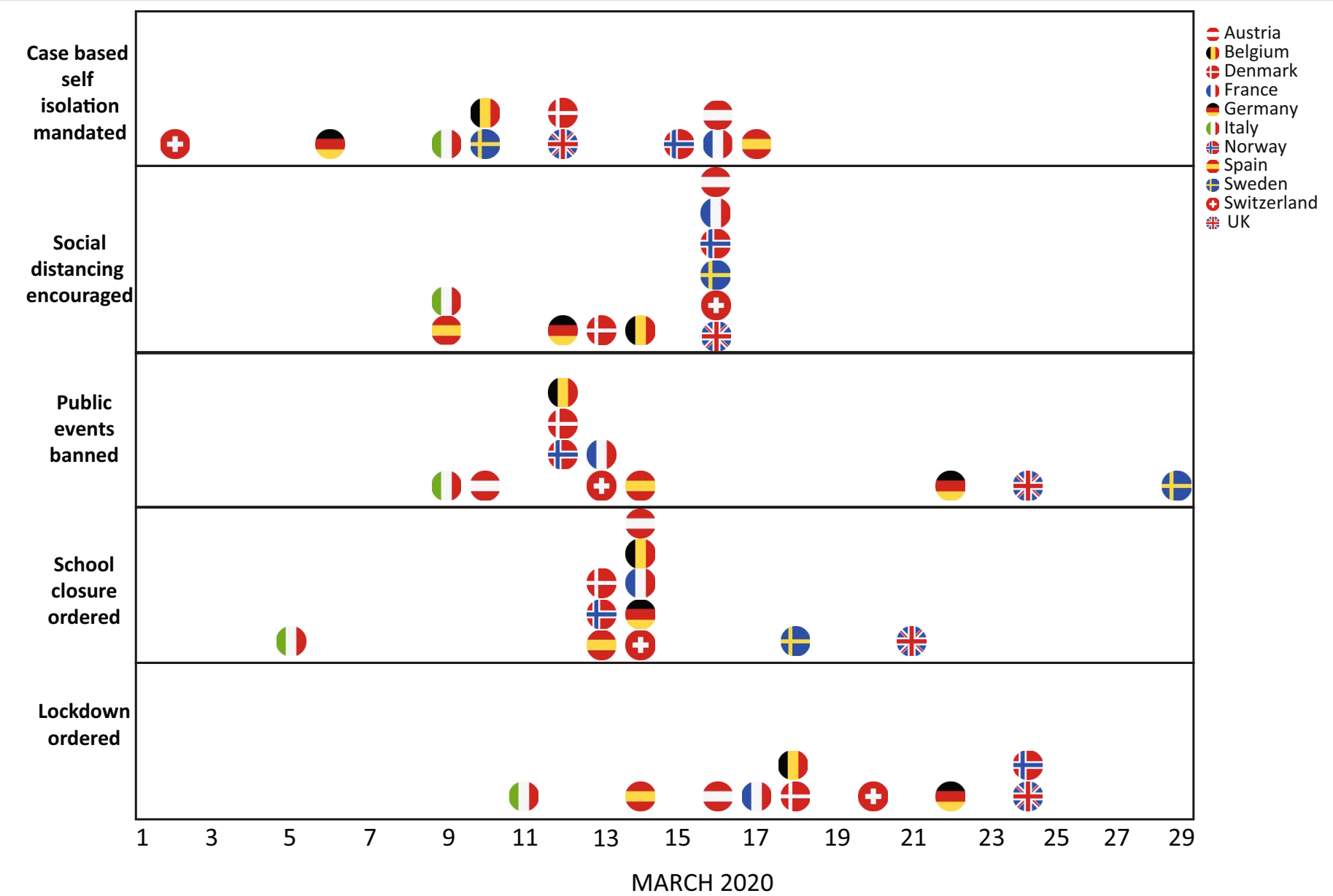

Extended Data Fig. 4 | Timings of interventions. Timings of interventions for the 11 European countries included in our analysis. For further details, see the Supplementary Information. 


\section{Article}

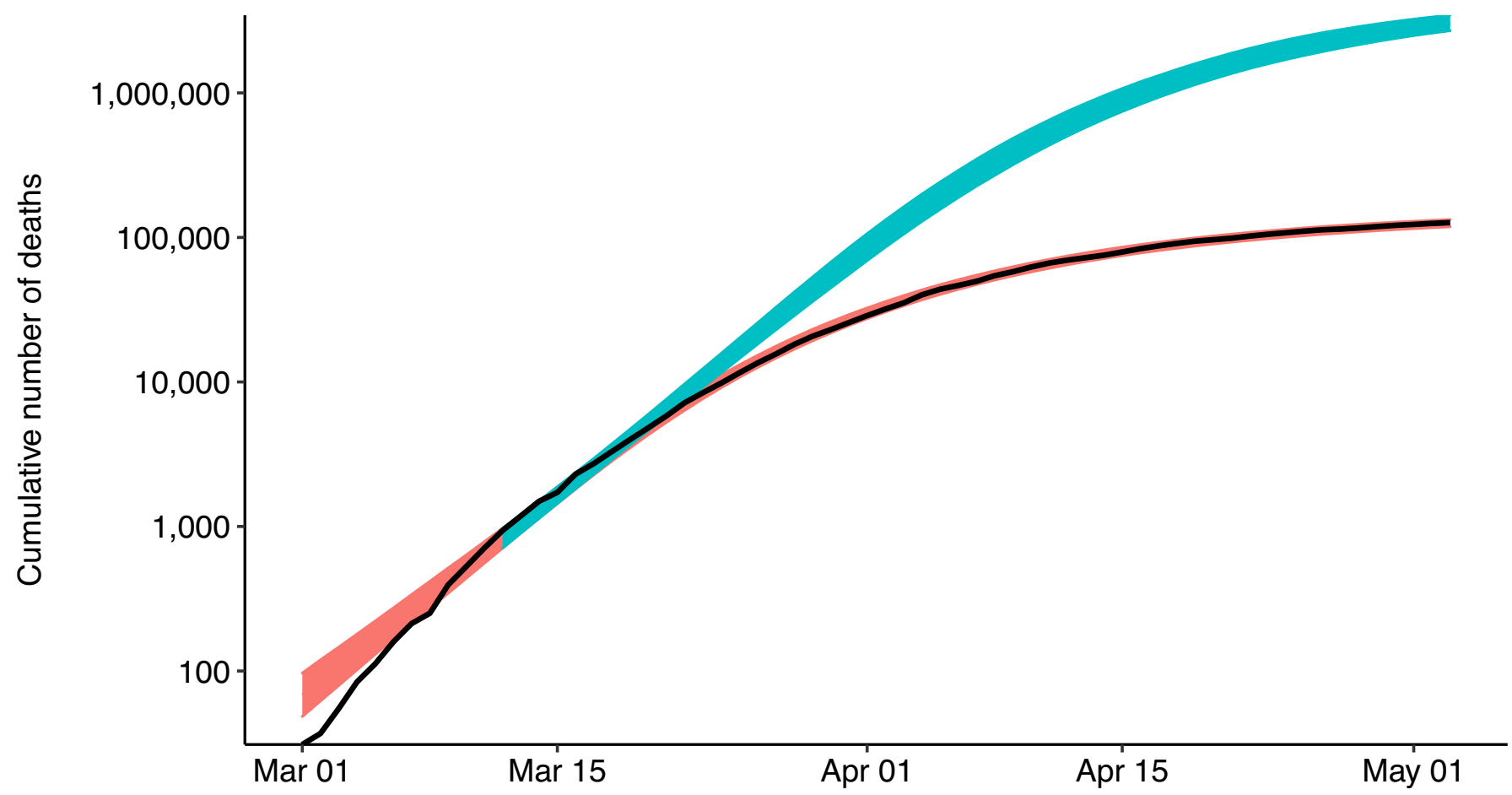

Extended Data Fig. 5 | Deaths averted owing to interventions. Lower and upper credible interval for the cumulative number of deaths for the 11 European countries in our model with interventions (pink) and the no-interventions counterfactual model (blue). Reported deaths are shown as a thick black line. 
Extended Data Table 1 | Total forecasted deaths since the beginning of the epidemic up to 4 May 2020 in our model and in a counterfactual model that assumes no interventions had taken place

Forecasted deaths since the beginning of the epidemic up to 4th May in our model vs. a counterfactual model assuming no interventions had taken place

\begin{tabular}{|c|c|c|c|c|}
\hline Country & $\begin{array}{l}\text { Observed } \\
\text { Deaths up to } \\
\text { 4th May } \\
\text { (observed) }\end{array}$ & $\begin{array}{l}\text { Model estimated deaths up } \\
\text { to 4th May }\end{array}$ & $\begin{array}{l}\text { Model estimated deaths up } \\
\text { to 4th May } \\
\text { (counterfactual model } \\
\text { assuming no interventions } \\
\text { have occurred) }\end{array}$ & $\begin{array}{l}\text { Model deaths averted up to } \\
\text { 4th May } \\
\text { (difference between } \\
\text { counterfactual and actual) }\end{array}$ \\
\hline Austria & 600 & $620[520-720]$ & $66,000[40,000-86,000]$ & $65,000[40,000-85,000]$ \\
\hline Belgium & 7,924 & $7,300[6,400-8,400]$ & $120,000[93,000-140,000]$ & $110,000[86,000-130,000]$ \\
\hline Denmark & 493 & $500[430-590]$ & $34,000[17,000-50,000]$ & $34,000[17,000-49,000]$ \\
\hline France & 25,201 & $23,000[21,000-27,000]$ & $720,000[590,000-850,000]$ & $690,000[570,000-820,000]$ \\
\hline Germany & 6,831 & $6,800[6,000-7,900]$ & $570,000[370,000-780,000]$ & $560,000[370,000-770,000]$ \\
\hline Italy & 29,079 & $31,000[27,000-35,000]$ & $670,000[540,000-800,000]$ & $630,000[510,000-760,000]$ \\
\hline Norway & 208 & $210[170-250]$ & $12,000[3,400-24,000]$ & $12,000[3,200-23,000]$ \\
\hline Spain & 25,613 & $25,000[22,000-28,000]$ & $470,000[390,000-560,000]$ & $450,000[360,000-540,000]$ \\
\hline Sweden & 2,769 & $2,800[2,500-3,300]$ & $28,000[15,000-49,000]$ & $26,000[12,000-46,000]$ \\
\hline Switzerland & 1,476 & $1,500[1,300-1,800]$ & $54,000[36,000-73,000]$ & $52,000[34,000-71,000]$ \\
\hline $\begin{array}{l}\text { United } \\
\text { Kingdom }\end{array}$ & 28,734 & $29,000[25,000-34,000]$ & $500,000[400,000-610,000]$ & $470,000[370,000-580,000]$ \\
\hline All & 128,928 & $\begin{array}{l}130,000 \\
{[120,000-140,000]}\end{array}$ & $\begin{array}{l}3,200,000 \\
{[2,900,000-3,600,000]}\end{array}$ & $\begin{array}{l}3,100,000 \\
{[2,800,000-3,500,000]}\end{array}$ \\
\hline
\end{tabular}




\section{Reporting Summary}

Nature Research wishes to improve the reproducibility of the work that we publish. This form provides structure for consistency and transparency in reporting. For further information on Nature Research policies, see our Editorial Policies and the Editorial Policy Checklist.

\section{Statistics}

For all statistical analyses, confirm that the following items are present in the figure legend, table legend, main text, or Methods section.

$\mathrm{n} / \mathrm{a} \mid$ Confirmed

$\square$ The exact sample size $(n)$ for each experimental group/condition, given as a discrete number and unit of measurement

$\square$ A statement on whether measurements were taken from distinct samples or whether the same sample was measured repeatedly

$\triangle$ The statistical test(s) used AND whether they are one- or two-sided

Only common tests should be described solely by name; describe more complex techniques in the Methods section.

$\bigotimes$ A description of all covariates tested

$\square$ A description of any assumptions or corrections, such as tests of normality and adjustment for multiple comparisons

$\square$ A full description of the statistical parameters including central tendency (e.g. means) or other basic estimates (e.g. regression coefficient)

AND variation (e.g. standard deviation) or associated estimates of uncertainty (e.g. confidence intervals)

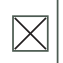

For null hypothesis testing, the test statistic (e.g. $F, t, r$ ) with confidence intervals, effect sizes, degrees of freedom and $P$ value noted

Give $P$ values as exact values whenever suitable.

$\square$ For Bayesian analysis, information on the choice of priors and Markov chain Monte Carlo settings

$\square \bigotimes$ For hierarchical and complex designs, identification of the appropriate level for tests and full reporting of outcomes

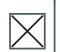
Estimates of effect sizes (e.g. Cohen's $d$, Pearson's $r$ ), indicating how they were calculated

Our web collection on statistics for biologists contains articles on many of the points above.

\section{Software and code}

Policy information about availability of computer code

Data collection No such software was used

Data analysis RStan version 2.19.3 was used within $\mathrm{R}$ version 3.6.3

For manuscripts utilizing custom algorithms or software that are central to the research but not yet described in published literature, software must be made available to editors and reviewers. We strongly encourage code deposition in a community repository (e.g. GitHub). See the Nature Research guidelines for submitting code \& software for further information.

\section{Data}

Policy information about availability of data

All manuscripts must include a data availability statement. This statement should provide the following information, where applicable:

- Accession codes, unique identifiers, or web links for publicly available datasets

- A list of figures that have associated raw data

- A description of any restrictions on data availability

All source code and data necessary for the replication of our results is available at https://github.com/ImperialCollegeLondon/covid19model The full set of posterior draws from our model are available at https://reshare.ukdataservice.ac.uk/854380/ 


\section{Field-specific reporting}

Please select the one below that is the best fit for your research. If you are not sure, read the appropriate sections before making your selection.

\ Life sciences

$\square$ Behavioural \& social sciences

Ecological, evolutionary \& environmental sciences

For a reference copy of the document with all sections, see nature.com/documents/nr-reporting-summary-flat.pdf

\section{Life sciences study design}

All studies must disclose on these points even when the disclosure is negative.

Sample size No samples were collected; data on the count of COVID-19-related deaths over time in 11 European countries was used.

Data exclusions No data was excluded.

Replication n/a

Randomization $\mathrm{n} / \mathrm{a}$

Blinding $\quad n / a$

\section{Reporting for specific materials, systems and methods}

We require information from authors about some types of materials, experimental systems and methods used in many studies. Here, indicate whether each material, system or method listed is relevant to your study. If you are not sure if a list item applies to your research, read the appropriate section before selecting a response.

Materials \& experimental systems

\begin{tabular}{|c|c|}
\hline $\mathrm{n} / \mathrm{a}$ & Involved in the study \\
\hline & $\square$ Antibodies \\
\hline $\mathbb{N}$ & Eukaryotic cell lines \\
\hline$\Delta$ & Palaeontology and archaeology \\
\hline$\nabla$ & $\square$ Animals and other organisms \\
\hline$k$ & $\square$ Human research participants \\
\hline Х & $\square$ Clinical data \\
\hline$凶$ & $\square$ Dual use research of concern \\
\hline
\end{tabular}

\begin{tabular}{l|l}
\multicolumn{2}{l}{ Methods } \\
\hline n/a & Involved in the study \\
$\searrow$ & $\square$ ChIP-seq \\
$\searrow$ & $\square$ Flow cytometry \\
$\searrow$ & $\square$ MRI-based neuroimaging
\end{tabular}

Х $\square$ MRI-based neuroimaging 